\title{
Publisher's Note: Stochastic feedback control of quantum transport to realize a dynamical ensemble of two nonorthogonal pure states [Phys. Rev. B 93, 085127 (2016)]
}

Shakib Daryanoosh, Howard M. Wiseman, and Tobias Brandes

(Received 25 February 2016; published 4 March 2016)

DOI: 10.1103/PhysRevB.93.119902

This paper was published online on 18 February 2016 with typographical errors on page 2 and 3. On page 2, right-hand column, the ninth line below Eq. (7) should read as "different choices of the parameters $\left\{\beta_{l}\right\},\left\{S_{m l}\right\}$ give rise to ...". On page 3, left-hand column, the sixth line below Eq. (8) should read as "it will be necessary to control the above parameters $\left\{\beta_{m}\right\},\left\{S_{m l}\right\} \ldots$..." The paper has been corrected as of 24 February 2016. The text is correct in the printed version of the journal. 